\title{
Isolation of rice allergenic cDNA clones from a rice cDNA library by immunoscreening with a polyclonal antibody specific to $16 \mathrm{kD}$ rice allergenic protein
}

\author{
Nam-il Kim, ${ }^{1}$ Woo-chang Kim, ${ }^{1}$ Sang-mi Lee, ${ }^{2}$ \\ Hee-kyeong Lee, ${ }^{1}$ Hae-ik Rhee, ${ }^{1}$ \\ Yong-soon Choi, ${ }^{1}$ Yeon-ho Jung ${ }^{1}$ \\ and Sang-hoon $\mathrm{Cha}^{1,3}$ \\ ${ }^{1}$ Kangwon National University, College of Agriculture \& \\ Life Sciences, Division of Food Sci. \& Biotechnology, \\ Chunchon 200-701, Korea \\ ${ }^{2}$ Yonsei University, College of Medicine, Department \\ of Parasitology, Seoul 120-752, Korea \\ ${ }^{3}$ Corresponding author: Tel, 82-361-250-6485; \\ Fax, 82-361-54-3835; E-mail, chash@kangwon.ac.kr
}

Accepted 7 October 1999

Abbreviations: RA, rice allergen; SDS-PAGE, Sodium dodecyl sulfate-polyacrylamide gel electrophoresis; IPTG, isopropyl- $\beta$-Dthiogalactopyranoside

\begin{abstract}
Clinical cases of type-1 hypersensitive reaction to rice (Oryza sativa) have been reported in western countries as well as in Japan. Among rice proteins, 14-16 kD globulin proteins encoded by multiple gene family have been identified as major rice allergens. In this study, a rice cDNA library was constructed using $\lambda$ UniZap vector and screened with a rat anti-16 kD globulin protein polyclonal antibody in order to isolate Korean rice allergenic CDNA clones. Five independent cDNA clones, termed RAK1-5, were obtained after second rounds of plaque assay and immunoblot analysis. These clones encoded 13$19 \mathrm{kD}$ recombinant proteins upon IPTG induction, which were identified by the polyclonal antibody in immunoblot analysis. DNA sequencing analysis showed that RAK1-4 have $99 \%$ sequence homology with RA5b, and RAK5 is closely related with RA14c. This result indicated that RA5b gene is widely distributed in our cDNA library among other possible rice allergenic genes, and more study is needed to isolate heterogeneous or novel rice allergen genes.
\end{abstract}

Keywords: rice, allergy, allergen, cDNA library

\section{Introduction}

Salt-soluble proteins in rice endosperm have been recognized by serum IgE of patients showing hypersensitive reaction to rice ingestion (Matsuda et al., 1988). Among rice proteins, $16 \mathrm{kD}$ protein has been known as one of the major allergens in rice grain, and it also inhibits serum $\lg \mathrm{E}$ binding to other cereal grain extracts including wheat, corn and millet (Urisu et al., 1991). These rice allergenic proteins are rice seed albumin and exhibit microheterogeneity in molecular weight (14-16 kD) (Matsuda et al., 1991).

In western countries, patients who have sensitized to rice are steadily increasing probably due to the increased consumption of oriental meals or more attentive medical care (Andre et al., 1994). It is also interesting to note that incidence of rice allergy is common in Japan, even though rice is ordinary meals. This suggests that rice allergy can be an important issue in public health in this country. In contrast to Japan, any report of rice allergy has not been documented in Korea where rice is generally consumed in every meal as in Japan. Clinical importance of rice allergy was demonstrated by the report that high RAST (radio-allergosorbent test) score for rice antigens has close relationship to the development of severe atopic dermatitis, rather than mite or egg white (Ikezewa et al., 1992a). In addition, treatment with hypoallergenic rice substantially reduced the clinical symptom of rice allergenic patients (Watanabe et al., 1990; lkezewa et al., 1992b).

At present, several rice allergens have been identified. A cDNA clone of rice allergen, RA17, was isolated from a rice (Oryza sativa L. var japonica cv Nipponbare) cDNA library using oligonucleotide probe, and turned out to be an $\alpha$-amylase/trypsin inhibitor family (Izumi et al., 1982). Other rice allergen (RA) cDNA clones have been identified by screening rice genomic or cDNA libraries by way of nucleic acid hybridization method using a radioactive-labeled RA17 DNA fragment (Adachi et al., 1993; Alvarez et al., 1995). In this study, we generated a rice cDNA library from Korean rice cultivar using $\lambda$ UniZap vector. This library was screened by immunological approach using a rat polyclonal antibody against $14-16 \mathrm{kD}$ rice proteins that immunologically cross-reactive each other.

\section{Materials and Methods}

Generation of a rat polyclonal antibody 
Salt-soluble rice proteins were obtained from rice seeds as described (Shibasaki et al., 1979) and 14-16 kD rice allergenic proteins were separated with Prep cell electrophoresis (BioRad, Richmond, CA). The resulting protein sample was dialyzed in phosphate buffered saline (PBS). Then, $1 \mathrm{mg}$ of the protein was mixed with Freund's complete adjuvant (Sigma Co., St. Louis, MO) and used to immunize three female Fisher rats by intraperitoneal route. Two successive booster injections were performed every other weeks and Freund's incomplete adjuvant (Sigma Co.) was used for booster injection. The rats were sacrificed one week after final injection and serum was harvested from peripheral blood.

\section{Construction of a rice cDNA library}

Messenger RNA was isolated from Korean II-poom rice cultivar using mRNA isolation kit (In vitrogen, San Diego, USA), and cDNA library was constructed with $\lambda$ UniZap vector kit (Stratagene, San Diego, USA) and in vitro packaging extract (Beringer Mannheim Biochemicals (BMB), Germany) according to manufacturer's protocols.

\section{Isolation of positive phage clones}

About 25,000 phages were mixed with $600 \mu \mathrm{l}$ of $\mathrm{XL}-1$ Blue MRF' cells (Stratagene) suspended in $10 \mathrm{mM} \mathrm{MgSO}_{4}$ at $O . D_{.600}=1.0$, and incubated at $37^{\circ} \mathrm{C}$ for $15 \mathrm{~min}$. Eight $\mathrm{ml}$ of top agar was added into the mixture and poured onto each LB plate $(150 \times 15 \mathrm{~mm})$. The LB plates were incubated at $37^{\circ} \mathrm{C}$ until plaques were visible, and nitrocellulose filters soaked in $10 \mathrm{mM}$ isopropyl- $\beta$-D-thiogalactopyranoside (IPTG) were overlaid onto the plates. The plates were incubated overnight at $32^{\circ} \mathrm{C}$. The filters were removed from the plate, washed in PBS with gentle scrubbing and blocked with $1 \%$ bovine serum albumin (BSA) (Sigma Co.) in PBS containing 0.05\% tween-20 (PBST) for $1 \mathrm{~h}$ at room temperature (RT) with rocking. After washing with PBST three times, the filters were incubated with a rat polyclonal antibody that previously absorbed with XL-Blue MRF cell lysate for $2 \mathrm{~h}$ at RT. The filters were washed again with PBST three times, and goat anti-rat $\lg G$ conjugated with alkaline phosphatase (AP) (Sigma Co.) that diluted at 1:2,000 in 1\% BSA solution was added. The filters were incubated for $1 \mathrm{~h}$ at RT. BCIP/NBT substrate (Amersham) was used to visualize the signal, and the reaction was stopped by washing the filters with $\mathrm{dH}_{2} \mathrm{O}$. Then, the positive plaques were cored out from the LB plates using sterile pasteur pipettes, and the phages were released by resuspending in $1 \mathrm{ml}$ SM buffer containing $40 \mu \mathrm{l}$ chloroform for the second round of immuno-screening as described above.

\section{Rescue of recombinant phagemids}

After second round of screening, phagemids from 9 positive $\lambda$ phage clones were rescued by in vivo excision. Briefly, $100 \mu \mathrm{l} \mathrm{XL-1}$ Blue MRF cells (O.D.600=1), $50 \mu \mathrm{l}$ phage resuspension and $1 \mu \mathrm{L}$ R 408 helper phage $\left(>10^{11}\right.$ $\mathrm{pfu} / \mathrm{ml}$ ) (Stratagene) were mixed and incubated at $37^{\circ} \mathrm{C}$ for $15 \mathrm{~min}$. Thence, $3 \mathrm{ml} 2 \times$ YT media was added into each tube, and incubated at $37^{\circ} \mathrm{C}$ for $3 \mathrm{~h}$ in the shaking incubator. $\lambda$ phages in the cultures were inactivated by incubating the culture tubes at $70^{\circ} \mathrm{C}$ for $20 \mathrm{~min}$, and the supernatants were harvested by centrifugation. The resulting phagemids in the supernatants were incubated with $\mathrm{XL}-1$ Blue MRF cells (Stratagene) at $37^{\circ} \mathrm{C}$ for $15 \mathrm{~min}$, and the mixtures were spread onto LB plates containing 50 $\mu \mathrm{g} / \mathrm{ml}$ ampicillin (LB-amp plate). After overnight incubation at $37^{\circ} \mathrm{C}$, E. coli colonies from each LB-amp plate were randomly picked, and plasmids were isolated using Qiagen Miniprep kit and treated with Xho I and Eco RI (BMB) in order to identify the size of CDNA inserts.

\section{Immunoblot analysis}

E. coli colonies were grown in $2 \mathrm{ml}$ LB-amp medium at $37^{\circ} \mathrm{C}$ until O.D.600 reaches about 0.8 , and expression of recombinant rice allergenic proteins was induced by adding $1 \mathrm{mM}$ IPTG for $4 \mathrm{~h}$. Thence, the cells were harvested by centrifugation and resuspended in $200 \mu \mathrm{l}$ SDS-PAGE sample buffer. The samples were boiled for $5 \mathrm{~min}$, the cellular proteins were separated by $12 \%$ SDS-PAGE, and transferred to nitrocellulose membrane (Amersham). The membrane was blocked with $1 \%$ BSA in PBST for $1 \mathrm{~h}$ at room temperature, and incubated with an immunized or normal rat sera that previously adsorbed with XL-1 Blue MRF cell lysate. The membrane was washed with PBST three times, and a goat anti-rat IgG conjugated with AP was added. BCIP/NBT substrate was used to visualize the signal.

\section{Automatic DNA sequencing}

Five rice cDNA clones showing positive signal in immunoblot were chosen for DNA sequencing analysis. Plasmids were isolated using Qiagen Miniprep kit, and polymerase chain reaction (PCR) was performed with cy5TM AutoCycleTM Sequencing Kit (Pharmacia Biotech) according to manufacturer's protocol. Sequencing gel was prepared using Long RangerTM Gel solution (FMC Corp.), and automatic DNA sequencing was performed using ALFexpress sequencers (Pharmacia Biotech).

\section{Results and Discussion}

\section{Construction and screening of a rice cDNA library}

cDNAs were synthesized using mRNA from rice (Oryza sativa) seed and ligated into $\lambda$ UniZap vector as described in the Materials \& Methods. The resulting rice cDNA library has $4.5 \times 10^{5}$ independent clones. The success of isolating allergens by immunoscreening of cDNA libraries using patient's IgE is strictly dependent upon the availability of patient's serum with high IgE titer. However, it is 
extremely difficult to get patient's serum with high titer of $\mathrm{IgE}$ because only trace level of $\mathrm{lgE}$ is present in sera.

Since there has not been any clinical case report for rice allergy in Korea, sera from patients with rice allergy were not available. Nonetheless, sera from ten Japanese rice allergic patients (kindly provided by Dr. M. Eric Gershwin, University of California at Davis, CA., USA) were obtained and tested for their $\lg E$ reactivity to rice proteins by western blot. Only one serum showed weak signal, but not still good enough to be used in cDNA library screening.

Therefore, we generated rat sera specific to $16 \mathrm{kD}$ rice allergenic protein and used this polyclonal antibody to screen the rice cDNA library as an alternative. The immuno-screening result showed that positive clones appeared about 6 out of 30,000 phage plaques $(0.02 \%)$ indicating that positive cDNA clones are relatively abundant in our library. Ten putative positive phage plaques were cored out, and second round of immunoscreening was carried out to confirm the positive reactivity of the phage plaques to the rat polyclonal antibody.

After the second screening, 9 phage plaques were finally obtained, and pBlueScript plasmids containing rice cDNA insert were rescued from the recombinant phages by in vivo excision. Thereafter, miniprep plasmid from nine clones was treated with Xho I and Eco RI in order to analyze the size of cDNA inserts by agarose gel electrophoresis. Xho I and Eco RI restriction endonucleases were used since these restriction sites have been utilized to clone rice cDNA fragments into $\lambda$ UniZap vector. Restriction fragment analysis shows that all nine clones have cDNA inserts of different length varied from 500-800 bp indicating that we might isolate different population of rice cDNAs (Figure 1).

\section{Immunoreactivity of RAK clones with polyclonal antibodies}

Nine E. coli colonies were cultured, and expression of

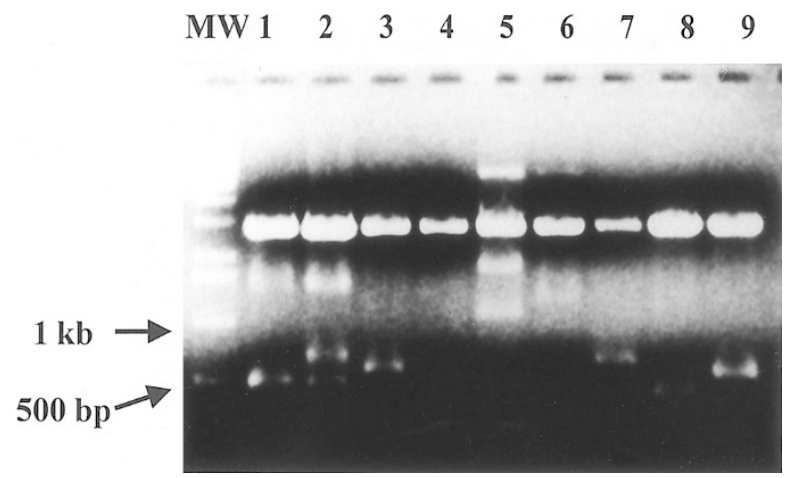

Figure 1. Identification of CDNA inserts by $1 \%$ agarose gel electrophoresis. After second round of screening, phagemids were rescued from nine $\lambda$ phage clones by in vivo excision using R408 helper phage. The phagemid DNA was isolated from E. coli culture and restricted with Xho I and Eco RI in order to identify the size of each rice allergenic CDNA insert. recombinant proteins encoded by rice cDNA inserts was induced by adding IPTG as described in the Materials and Methods. Thence, total E. coli proteins were separated by $12 \%$ SDS-PAGE, and immunoreactivity of recombinant proteins to anti-16 $\mathrm{kD}$ rat sera was analyzed by immunoblot (Figure 2). The data showed that 5 out of 9 clones expressed recombinant proteins which showed prominent reactivity to anti-16 $\mathrm{kD}$ rat sera ranging $13-19$ $\mathrm{kD}$ in size. These five clones were termed RAK 1-5 (Figure 2 lane 1, 3, 4, 5 and 9, respectively). Unimmunized negative control rat sera did not show any reactivity to the recombinant proteins (data not shown).

\section{DNA sequence analysis of RAKs}

Rice allergenic cDNA clones (RA17) were originally isolated by screening a rice cDNA library using an oligonucleotide probe based upon peptide sequence of rice allergenic protein (Izumi et al., 1982). These rice allergenic cDNAs encode the amylase/typsin inhibitor family and turn out to be a member of multigene family. In order to isolate more rice allergens, a rice cDNA library was screened by hybridization with a 370 bp ${ }^{32} \mathrm{P}$-labeled RA17 cDNA probe, and closely related rice allergen cDNAs, RA5b \& RA14b, were isolated (Alvarez et al., 1995). In this study, we screened the rice cDNA library using a rat anti-16 kD

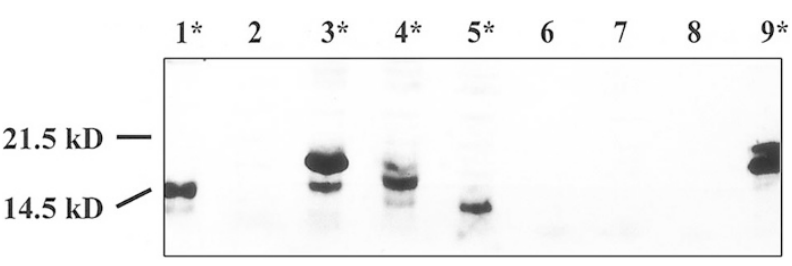

Figure 2. Analysis of recombinant rice allergenic proteins by $12 \%$ SDSPAGE and immunoblot. E. coli colonies (lane 1-9) were grown in LB-amp medium, and expression of recombinant rice allergenic proteins was induced by adding IPTG in the culture. Thence, the cellular proteins were separated by $12 \%$ SDS-PAGE. For immunoblot, the nitrocellulose membranes were incubated with polyclonal rat sera. Asterisks indicate the phagemid clones expressing the recombinant proteins that specifically recognized by anti- $16 \mathrm{kD}$ polyclonal antibody.

Table 1. Percentage of nucleotide and amino acid sequence homologies between RA5b and RAK1-4

\begin{tabular}{cccccc}
\hline & RA5b & RAK1 & RAK2 & RAK3 & RAK4 \\
\hline RA5b & $100 \%$ & $99.0 \%$ & $98.7 \%$ & $98.9 \%$ & $98.3 \%$ \\
& $(100 \%)$ & $(98.1 \%)^{*}$ & $(96.3 \%)$ & $(98.3 \%)$ & $(97.9 \%)$ \\
RAK1 & - & $100 \%$ & $100 \%$ & $99.4 \%$ & $99.0 \%$ \\
& & $(100 \%)$ & $(100 \%)$ & $(97.1 \%)$ & $(97.9 \%)$ \\
RAK2 & - & - & $100 \%$ & $99.2 \%$ & $99.0 \%$ \\
& & & $(100 \%)$ & $(95.7 \%)$ & $(97.9 \%)$ \\
RAK3 & - & - & - & $100 \%$ & $99.0 \%$ \\
& & & & $(100 \%)$ & $(96.9 \%)$ \\
RAK4 & - & - & - & - & $100 \%$ \\
& & & & & $(100 \%)$ \\
\hline
\end{tabular}

*Parenthesis shows amino acid sequence homology. 


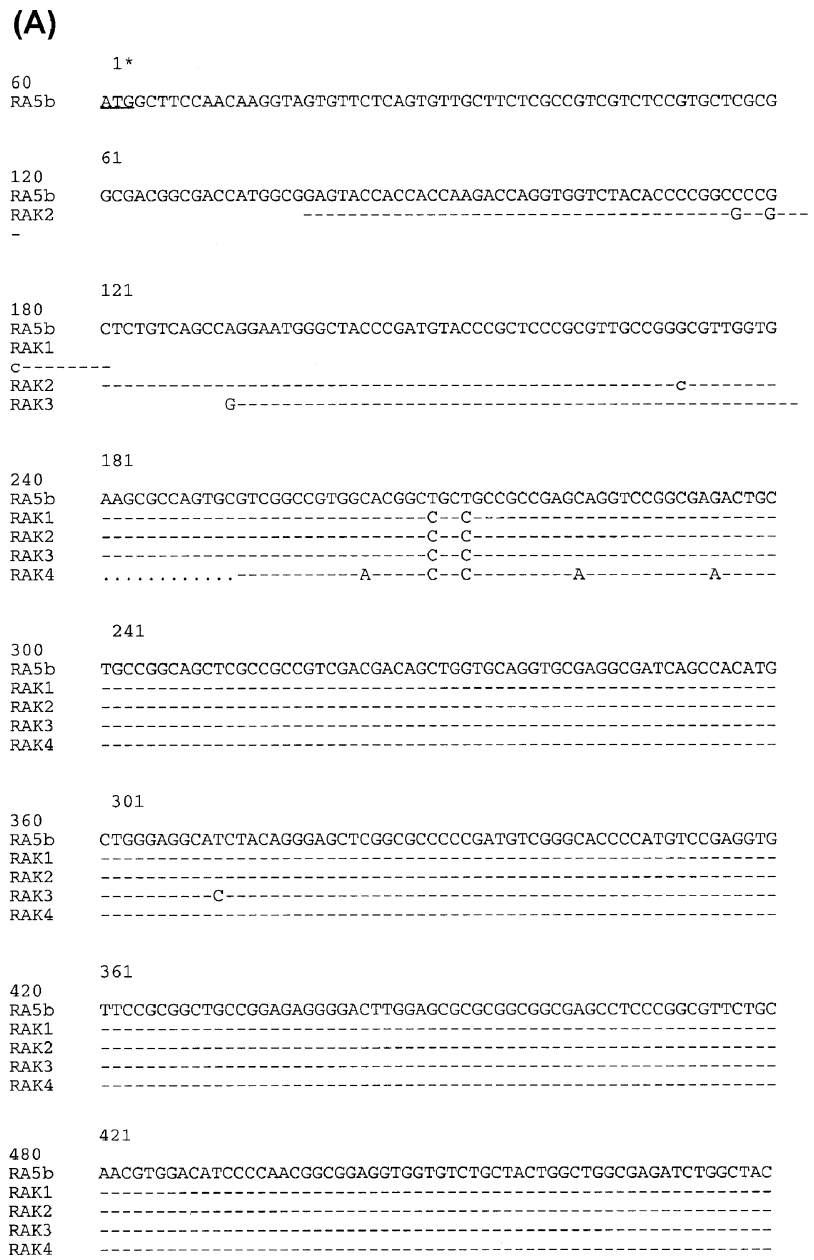

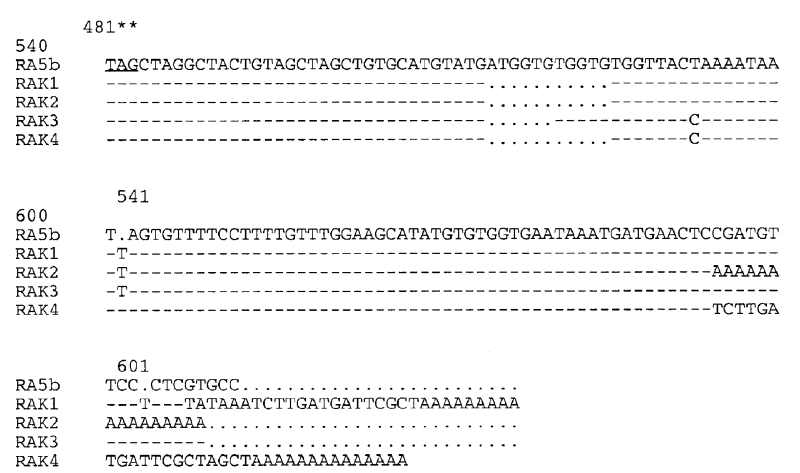

\section{(B)}

$$
\begin{aligned}
& \text { RA5 } \\
& \text { RAK } \\
& \text { RAK } 3
\end{aligned}
$$

$\frac{1}{\text { MASNKVVF SVLLLAVVSVLAATATMAEYHHQDQVVYTPAPLCQPGMGYPM }}$

RA5B Y YPLPRCRALVKRQCVGRGTAAAAEQVRRDCCRQLAAVDDSWCRCEAISHM

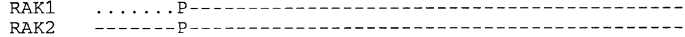

RAK3

RAK

101

RA5b
RAK1
RAK2
RAK3

RAKA

RA5b
RAK1
RAK2
RAK3

RAK4

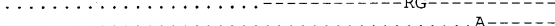

(1).-P

...........

101

LGGIYRELGAPDVGHPMSEVFRGCRRGDLERAAASLPAFCNVDI PNGGGG

T- -

151

VCYWLARSGY

(15)

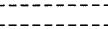

Figure 3. Comparison of nucleotide (A) and amino acid (B) sequences between RA5b and RAK1-4 ( $\cdots$ indicates no corresponding nucleotide or amino acid, --- indicates the same nucleotide or amino acid as above).

rice globulin sera. Five positive phage clones (RAK1-5) were finally obtained, and DNA sequence of rice CDNA was analyzed with previously known rice allergen cDNAs. The result shows that RAK1-4 are almost identical to RA5b (about 99\% homology at nucleotide level in the coding region and $96.3-99 \%$ homology at amino acid level) (Figure 3A \& B). RAK1 and RAK2 are the same gene except that RAK1 has more truncation at the 5 ' end. On the other hand, RAK5 has about $95.5 \%$ homology with RA14c at the nucleotide level suggesting that there is little rice strain-specific DNA variations among RA14 family (Figure 4A \& B).

In immunoblot using rice total protein and the rat polyclonal antibody, we always observe the presence of a cross-reactive protein of $30 \mathrm{kD}$ with unknown identity (data not shown). Unfortunately, we couldn't isolate any novel rice allergenic genes corresponding to the molecular weight above and may need more extensive screening. Although we characterized only 5 clones, it seems likely that RA5b gene is either the most frequent rice allergen or present dominantly in our particular cDNA library.
Treatment of rice allergenic patients with enzymatically cleaved hypoallergenic rice showed profound improvement of clinical symptoms (Watanabe et al., 1990; lkezewa et al., 1992b). However, generation of hypoallergenic rice by enzymatic treatment of rice seeds is cost inefficient and difficult for mass production. Thereby, development of new hypoallergic rice strains as a functional food have been attempted using classical approach such as isolation of rice cultivars that contain low content of 16

Table 2. Percentage of nucleotide and amino acid sequence homologies among RA14b, RA14c and RAK5

\begin{tabular}{cccc}
\hline & RA14b & RAK5 & RA14c \\
\hline RA14b & $100 \%$ & $95.0 \%$ & $96.7 \%$ \\
& $(100 \%)^{*}$ & $(87.0 \%)$ & $(91.2 \%)$ \\
RAK5 & - & $100 \%$ & $95.5 \%$ \\
& & $(100 \%)$ & $(92.9 \%)$ \\
RA14c & - & - & $100 \%$ \\
& & & $(100 \%)$ \\
\hline
\end{tabular}

*Parenthesis shows amino acid sequence homology. 
(A)

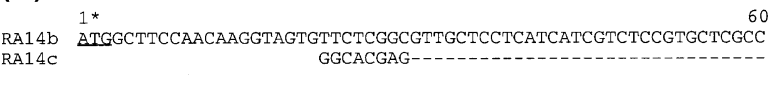
RA14b ${ }^{61}$ GCGACGGGACCCATGGCGGACCACCACAAAGACCAGGTGGTGTACAGCCTCGGCGAGCGT RA14b GCGACGGGACCCATGGCGGACCACCACAAAGACCAGGTGGTGTACAGCCTCGGCGAGCGT RA14C
RAK5 121 121
RA14b TGTCAGCCAGGAATGGGCTACCCGATGTACTCGCTGCCACGCTGCCGGGCGGTGGTGAAG RA14b TGTCAGCCAGGAATGGGCTACCCGATGTACTCGCTGCCACGCTGCCGGGCGGTGGTGAAG
RA14c
RAK5 181240 RA14C 241 300

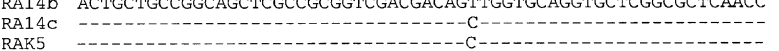
301360 RA14b ACATGGTTGGAGGCATCTACAGGGAGCTCGGCGCCACCGATGTTGGGCACCCCATGGCCG RA14c RAK5 3612420 RA14b AGGTGTTCCCCGGCTGCCGGAG RA14C - RAK5 421
RA14b TCTGCAACGTGGACATCCCCAATGGGACAGGTGGTGTCTGCTACTGGCTAGGTTAT. . . C RA14C - RAK5 -

481 ** 540 RA14b CTAGGACCCCAGAACTGGTCACTAGGCTACTAAAGCTAGCTGTGTGTATGACTCTGTGG RA14C - $\mathrm{RAK5}$ 541600 RA14b GGTTGCTAAATAACTAGTGCTTTCATTTGTCAGGAAGCATATATACATATGGTGAATAAA RA14c RAK5

(B)

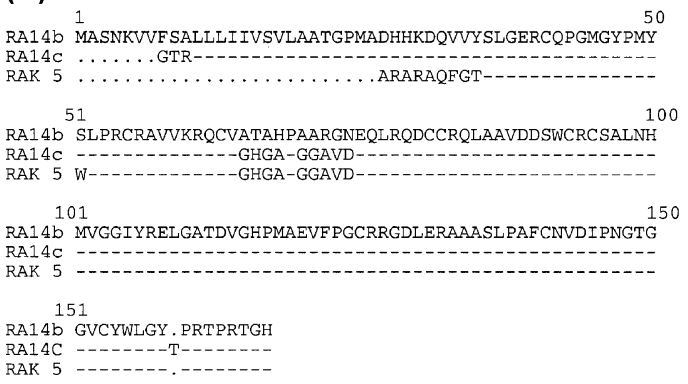

Figure 4. Comparison of nucleotide (A) and amino acid (B) sequences between RA14b and RAK5 ( $\cdots$ indicates no corresponding nucleotide or amino acid, --- indicates the same nucleotide or amino acid as above).

$\mathrm{kD}$ allergenic protein from gamma-ray induced mutant rice (Nishio \& lida, 1993). In addition, molecular and genetic approach by introducing an antisense gene to generate transgenic rice plants has been reported (Tada et al., 1996).

We believe that rice allergenic cDNA clones obtained in this study can be applied in genetic alteration of rice as mentioned above and provision of genetic resources from Korean rice cultivar.

\section{Acknowledgment}

This study was supported by a grant (No. 295201-3) from R\&D Promotion Center, Ministry of Agriculture and Fishery, Korea.

\section{References}

Adachi, T., Izumi, H., Yamada, T., Tanaka, K., Takeuchi, S., Nakanuma, R. and Matsuda, T. (1993) Gene structure and expression of rice seed allergenic proteins belonging to the $\alpha$ amylase/trypsin inhibitor gene family. Plant Mol. Biol. 21: 239248

Alvarez, A. M., Adachi, T., Nakase, M., Aoki, N., Nakanuma, R. and Matsuda, T. (1995) Classification of rice allergenic protein cDNAs beloninging to the a-amylase/trypsin inhibitor gene family. Biochimica Biophysica Acta 1251: 201-204

Andre, F., Andre, C., Colin, L., Cacaraci, F. and Cavagna, S. (1994) Role of new allergens and of allergen consumption in the increased incidence of food sensitizations in France. Toxicol. 93: 77-83

Ikezawa, Z., Miyakawa, K., Komatsu, H., Suga, C., Miyakawa, J., Sugiyama, A., Sasaki, T., Nakajima, H., Hirai, Y. and Suzuki, Y. (1992a) A probable involvement of rice allergy in severe type of atopic dermatitis in Japan. Acta Derm Venereol Suppl (Stockh) 176: 103-107

Ikezawa, Z., Ikebe, T., Ogura, H., Odajima, H., Kurosaka, F., Komatsu, H., Sase, K., Suga, C., Sugiuchi, M. and Suguro, H. (1992b) Mass trial of hypoallergenic rice (HRS-1) produced by enzymatic digestion in atopic dermatitis with suspected rice allergy. HRS-1 research Group. Acta Derm Venereol Suppl (Stockh) 176: 108-112

Izumi, H., Adachi, T., Fujii, N., Matsuda, T., Nakanuma, R., Tanaka, K., Urisu, A. and Kurosawa, Y. (1982) Nucleotide sequence of a cDNA encoding a major allergenic protein in rice seeds - Homology of the deduced amino acid sequence with members of a-amylase/trypsin inhibitor family. FEBS Lett. 302: $213-216$

Matsuda, T., Sugiyama, M., Nakanuma, R. and Torii, S. (1988) Purification and properties of an allergenic protein in rice grain. Agric. Biol. Chem. 52: 1465-1470

Matsuda, T., Nomura, R., Sugiyama, M. and Nakanuma, R. (1991) Immunochemical studies on rice allergenic proteins. Agric. Biol. Chem. 55: 509-513

Nishio, T. and lida, S. (1993) Mutants having a low content of $16-k D$ allergenic protein in rice (Oryza sativa L.). Theor. Appl. Genet. 86: 317-321

Shibasaki, M., Suzuki, S., Nemoto, H. and Kuroume, T. (1979) Allergenicity and lymphocyte-stimulating property of rice protein. J. Allergy Clin. Immunol. 64: 259-265

Tada, Y., Nakase, M., Adachi, T., Nakamura, R., Shimada, H., 
Takahashi, M., Fujimura, T. and Matsuda, T. (1996) Reduction of 14-16 kD allergenic proteins in transgenic rice plants by antisense gene. FEBS Lett. 391: 341-345

Urisu, A., Yamada, K., Masuda, S., Komada, H., Wada, E., Kondo, Y., Horiba, F., Tsuruta, M., Yasaki, T. and Yamada, M. (1991) 16-kilodalton rice protein is one of the major allergens in rice grain extract and reponsible for cross-allergenicity between cereal grains in the Poaceae family. Int. Arch. Allergy Appl. Immunol. 96: 244-252

Watanabe, M., Miyakawa, J., Ikezawa, Z., Suzuki, Y., Hirano, T., Yoshizawa, T. and Arai, S. (1990) Production of hypoallergenic rice by enzymatic decomposition of constituent proteins. J. Food Sci. 55: 781-783 